WSU-HEP-0804

\title{
A note on the black hole information paradox in de Sitter spacetimes
}

\author{
Cosimo Bambi \\ Department of Physics and Astronomy, Wayne State University, Detroit, MI 48201, USA \\ Michigan Center for Theoretical Physics, University of Michigan, Ann Arbor, MI 48109, USA
}

(Dated: November 5, 2018)

\begin{abstract}
The possibility of stable or quasi-stable Planck mass black hole remnants as solution to the black hole information paradox is commonly believed phenomenologically unacceptable. Since we need a black hole remnant for every possible initial state, the number of remnants is expected to be infinite and that would lead to remnant pair production in any physical process with a total available energy roughly exceeding the Planck mass. In this note I point out that a positive cosmological constant of the Universe would naturally lead to an upper bound on the number of possible remnants.
\end{abstract}

PACS numbers: 04.70.Dy

\section{INTRODUCTION}

The Black Hole $(\mathrm{BH})$ information paradox is a well known issue in theoretical physics [1, 2]. In quantum mechanics, the evolution operator $U$ is unitary, i.e. $U^{\dagger} U=1$, which implies that a pure state must evolve into another pure state. On the other hand, BHs have a temperature and emit radiation via Hawking effect [3]. This radiation is almost thermal and thus does not depend on the features of the initial state, but only on the $\mathrm{BH}$ geometry outside the horizon ( $\mathrm{BH}$ mass, electric charge and angular momentum). In other words, the outgoing radiation does not carry any information about the initial state which collapsed into the BH. As the $\mathrm{BH}$ emits radiation, it loses mass. The crucial question is what happens at the end: if the $\mathrm{BH}$ evaporates completely and the final product is only thermal radiation, information is lost forever. In this case, a pure state would evolve into a mixed one, i.e. its evolution would be non-unitary. There are basically three answers to the question on the fate of the initial information: $i$ ) the evolution is indeed non-unitary, $i i)$ the radiation is not thermal and carries information, iii) information is stored in a long-lived or stable $\mathrm{BH}$ remnant. All the proposals, however, have some problems.

The possibility that information is lost and unitarity is violated was originally put forward in ref. [4]. In this case, the basic ingredients of quantum mechanics must be revised, in order to allow for a pure state to evolve into a mixed one. In particular, the unitary $\mathrm{S}$-matrix of quantum field theory, which connects any initial quantum state to its final quantum state, $\left|\psi_{\text {out }}\right\rangle=S\left|\psi_{\text {in }}\right\rangle$, is replaced by the non-unitary super-scattering operator $\$$, which maps an initial density matrix into a final density matrix

$$
\rho_{\text {out }}=\$ \rho_{\text {in }}
$$

and mixes quantum states. The problem is that energy conservation is violated and that there is no empty vacuum as ground state, which means we should live in a Universe similar to a thermal bath at the Planck temperature [5].
The second possibility is that information is encoded in the outgoing radiation [6] and unitarity is conserved. Such an idea relies on possible higher order effects which are not taken into account in the standard calculations. Here we need new physics which is radically different from the one we know and "sacred" concepts such as the ones of locality and causality must be abandoned. At present, this is likely the most appealing possibility, even because supported by the AdS/CFT conjecture. Nevertheless, one could expect that departure from standard calculations becomes important only when the $\mathrm{BH}$ approaches the Planck mass: for large $\mathrm{BHs}$, the radiation would be well described by the semi-classical framework, while, for small BHs, new physics would play and important role and the emitted radiation could carry all the information about the initial state. However, general arguments suggest that it is unlikely a sudden emission of the information in the last stages of evaporation [7]. The $\mathrm{BH}$ should emit about $N \sim M^{2} / M_{P l}^{2}$ quanta with average wavelength $\lambda \sim N / M_{P l}$, where $M$ is the initial $\mathrm{BH}$ mass and $M_{P l}$ is the Planck one. On the other hand, the size of the $\mathrm{BH}$ at this stage is about $1 / M_{P l}$, which means that the wave-function overlap is $1 / N^{3}$ and the simultaneous emission of $N$ quanta would be suppressed by the factor $1 / N^{3 N}$. Even in the case of a gradual emission of $N$ quanta, the final process would require a very long time, at the level of $N^{4} / M_{P l}$. So, the end product of Hawking evaporation would be a long-lived remnant, which is the last option.

Information is stored into a stable or quasi-stable remnant [7]. This possibility is suggested by the idea that semi-classical calculations of $\mathrm{BH}$ evaporation hold till the $\mathrm{BH}$ approaches the Planck mass, when quantum gravity and back reaction effects can no longer be neglected. Here the problem is that an infinite number of initial states implies an infinite number of remnants. Even assuming a tiny coupling constant between remnants and ordinary matter, virtual remnant states should affect all the quantum processes. Remnant pair production should be the result of any reaction with a total available energy exceeding the remnant mass [8]: for macroscopic processes, this is easy to achieve and, even if the remnant production probability is extremely small, mainly 
because the energy densities are always small, the existence of infinite states would be anyway catastrophic.

\section{UPPER BOUND ON THE NUMBER OF REMNANTS}

The argument against BH remnants is that an infinite number of them would be phenomenologically unacceptable [8]. Every initial state must be associated with a remnant and if we think that the number of initial states is infinite, the number of remnants must be infinite too.

The aim of this note is to show that a positive cosmological constant $\Lambda$ can provide a natural upper bound on the number of remnants. Cosmological and astrophysical evidences suggest that in the Universe $\Lambda>0[9]$. If this were the case, the Universe would be asymptotically de Sitter and we could estimate the corresponding entropy. For an an empty de Sitter spacetime with a cosmological constant equal to the one we can deduce from observations, the entropy is ${ }^{1}$

$$
S_{0}=\frac{3 \pi M_{P l}^{2}}{\Lambda} \sim 2 \cdot 10^{122} .
$$

In ref. [10] was conjectured that $S_{0}$ is roughly the logarithm of the total number of quantum states necessary to describe the Universe and therefore the direct count of the number of its degrees of freedom. Following this interpretation, the total number $\mathcal{N}$ of possible initial states which can collapse into a BH is bounded by

$$
\mathcal{N}<\mathcal{N}_{\text {bound }}=\exp \left(\frac{3 \pi M_{P l}^{2}}{\Lambda}\right) \sim 10^{10^{122}}
$$

Let us note that this is only an upper bound and that actually the number of remnants may be even much smaller.

One could also be worried about the fact that a huge amount of information is contained in an object with a Planck volume. This is not a fundamental problem, because the spacetime is curved and a small volume for an external observer could be a large or infinite volume inside the $\mathrm{BH}[2]$. An intuitive picture of how this is possible is sketched in fig. 1,

\section{DANGEROUS PROCESSES}

Even if there is not yet any reliable theory in which one can discuss quantum gravity objects like $\mathrm{BH}$ remnants, we can anyway try to estimate some implications of remnants from "reasonable" arguments.

\footnotetext{
${ }^{1}$ In the case of non-empty de Sitter spacetime, the entropy is smaller, so eq. (2) can be seen as an upper bound.
}

For example, it is common belief that we can take quantum gravity effects into account in low-energy particle physics by using an effective field theory, with nonrenormalizable and Planck mass suppressed operators. Here virtual BH remnants could induce baryon and lepton number violations and, in particular, proton decay [11]. In $3+1$ dimensions, proton decay would be described by the dimension six operator

$$
\mathcal{O}_{6} \sim \frac{\psi \psi \psi \psi}{M_{P l}^{2}}
$$

since two quarks of the proton are converted into a quark (or an anti-quark) and a charged lepton. The usual estimate of the proton lifetime is

$$
\tau_{p} \sim \frac{M_{P l}^{4}}{m_{p}^{5}} \sim 10^{45} \mathrm{yr}
$$

and has to be compared with present experimental limits, where several channels are bounded by $\tau_{p}>10^{33} \mathrm{yr}[12$ ]. If remnants exist, every remnant intermediate state is equally probable and integrating over an infinite number of states the decay width goes to infinity. In our case their number is instead finite and the proton lifetime is

$$
\tau_{p} \gtrsim \frac{1}{g^{2} \mathcal{N}_{\text {bound }}} \frac{M_{P l}^{4}}{m_{p}^{5}},
$$

where $g$ is the dimensionless coupling constant between ordinary matter and $\mathrm{BH}$ remnants which we put in front of the dimension six operator in eq. (44). If the number of remnants is not much smaller than the upper bound in eq. (3), the coupling constant $g$ has to be really small. However, the possibility of a tiny coupling constant cannot be rejected, because this is just an effective theory. There could be indeed mechanisms capable of suppressing the process. A proposal to get a small coupling can be found, for example, in ref. [13].

As for the remnant pair production in macroscopic physical processes, the estimate is even more difficult, albeit the phenomenon is based on more solid arguments. Here we have to expect some suppression factor due to the ratio between the remnant external volume and the macroscopic volume which provides the necessary amount of energy to create the remnant pair. Anyway, the problem still reduces to find a small coupling constant between $\mathrm{BH}$ remnants and ordinary matter.

Very dangerous processes may be represented by gravitational phenomena, because the validity of the Equivalence Principle requires that all the form of energy couples to gravity with the same strength. For example, let us consider the $10^{6}$ Solar mass BH which is believed to reside at the center of the Galaxy. Like any $\mathrm{BH}$, it is expected to emit Hawking radiation. For a Schwarzschild $\mathrm{BH}$ of mass $M$, the temperature $T$ is

$$
T=\frac{1}{8 \pi G_{N} M}=5 \cdot 10^{-18}\left(\frac{10^{6} M_{\odot}}{M}\right) \mathrm{eV}
$$




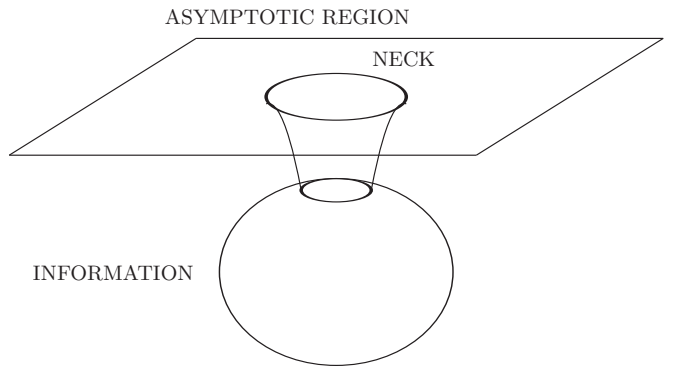

FIG. 1: Information could be stored within a large volume that appears of Planck size for an external observer living in the asymptotic region.

and is so low that it seems to be impossible to observe, even in the future. If remnants behave as ordinary particles, their emission would be Boltzmann suppressed by a huge Planck mass to $\mathrm{BH}$ temperature ratio, $\exp \left(-\frac{M_{P l}}{T}\right) \sim 10^{-10^{45}}$. If $\mathcal{N} \sim \mathcal{N}_{\text {bound }}$, they should anyway cause a sudden evaporation of the $\mathrm{BH}$ at the center of the Galaxy. However, such argument is weak, because the formation of remnants at the horizon may be more strongly suppressed: remnants may not be "elementary" and, as we do not expect that the emission of hydrogen atoms is similar to the one of protons, we can argue that the same holds for remnants.

\section{CONCLUSIONS}

The black hole information paradox is an open issue which clearly shows the difficulties to unify quantum me- chanics and gravity in a unique framework. Several proposal have been suggested, but all of them present serious problems.

If we believe that semi-classical calculations are reliable as far as the mass of the black hole is much larger than the Planck one, the most natural solution to the puzzle is that information is stored in stable or long-lived remnants. It is usually believed that there must exist an infinite number of remnants, one for every possible initial state, and this turns out to be phenomenologically unacceptable [8]. In this note I showed that the number of remnants could instead be finite and I provided an upper bound. The presence of a positive cosmological constant would constrain the number of degrees of freedom of the Universe and such a constraint can be seen at least as an upper bound on the number of initial states which can collapse into a black hole. That would make the number of remnants finite. So, the main argument against remnants may not exist. If the number of remnants is close to the upper bound, it is still difficult to assert if they are phenomenologically acceptable. For sure, they cannot be completely excluded as solution to the information black hole paradox, because we do not how to describe them and, in the framework of a low-energy effective field theory, they may be associated with very small coupling constants.

\section{Acknowledgments}

I wish to thank Katherine Freese for kind hospitality at the Michigan Center for Theoretical Physics, where this work was being written. The work is supported in part by NSF under grant PHY-0547794 and by DOE under contract DE-FG02-96ER41005.
[1] J. Preskill, arXiv:hep-th/9209058;

D. N. Page, arXiv:hep-th/9305040,

S. B. Giddings, arXiv:hep-th/9508151

L. Thorlacius, arXiv:hep-th/0404098

[2] S. B. Giddings, Phys. Rev. D 46, 1347 (1992) arXiv:hep-th/9203059.

[3] S. W. Hawking, Commun. Math. Phys. 43, 199 (1975) [Erratum-ibid. 46, 206 (1976)].

[4] S. W. Hawking, Phys. Rev. D 14, 2460 (1976);

S. W. Hawking, Commun. Math. Phys. 87, 395 (1982).

[5] D. J. Gross, Nucl. Phys. B 236, 349 (1984);

J. R. Ellis, J. S. Hagelin, D. V. Nanopoulos and M. Srednicki, Nucl. Phys. B 241, 381 (1984);

T. Banks, L. Susskind and M. E. Peskin, Nucl. Phys. B 244, 125 (1984);

A. Strominger, arXiv:hep-th/9410187

[6] D. N. Page, Phys. Rev. Lett. 44, 301 (1980);

G. 't Hooft, Nucl. Phys. B 256, 727 (1985).

[7] Y. Aharonov, A. Casher and S. Nussinov, Phys. Lett. B 191, 51 (1987).
[8] S. B. Giddings, Phys. Rev. D 49, 947 (1994) arXiv:hep-th/9304027;

S. B. Giddings, Phys. Rev. D 51, 6860 (1995) arXiv:hep-th/9412159.

[9] A. G. Riess et al. [Supernova Search Team Collaboration], Astron. J. 116, 1009 (1998) arXiv:astro-ph/9805201;

S. Perlmutter et al. [Supernova Cosmology Project Collaboration], Astrophys. J. 517, $565 \quad$ (1999) arXiv:astro-ph/9812133;

D. N. Spergel et al. [WMAP Collaboration], Astrophys. J. Suppl. 148, 175 (2003) arXiv:astro-ph/0302209.

[10] T. Banks, arXiv:hep-th/0007146

W. Fischler, Taking de Sitter seriously, talk given at Role of Scaling Laws in Physics and Biology - Celebrating the 60th Birthday of Geoffrey West, Santa Fe, USA, 2000.

[11] Y. B. Zeldovich, Phys. Lett. A 59, 254 (1976); S. W. Hawking, D. N. Page and C. N. Pope, Phys. Lett. B 86, 175 (1979);

C. Bambi, A. D. Dolgov and K. Freese, Nucl. Phys. B 
763, 91 (2007) arXiv:hep-ph/0606321;

C. Bambi, arXiv:0710.0235 [hep-ph]; C. Bambi and K. Freese, Class. Quantum Grav. 25, 195013 (2008) arXiv:0803.0749 [hep-th]].
[12] W. M. Yao et al. [Particle Data Group], J. Phys. G 33, 1 (2006).

[13] A. Casher and N. Raz, arXiv:0705.0444 [hep-th]. 